\title{
Irradiation of lung and esophagus tumors: A comparison of dose distributions calculated by anisotropic analytical algorithm and pencil beam convolution algorithm, a retrospective dosimetric study
}

\author{
Emmanuel Amankwaa-Frempong1, Frederik Vernimmen', Samuel Nguah Blay², Ramalingam Ezhilalan ${ }^{1}$ \\ ${ }^{I}$ Department of Radiotherapy, Cork University Hospital, Ireland. \\ ${ }^{2}$ Directorate of Child Health, Komfo Anokye Teaching Hospital, Kumasi, Ghana.
}

Received January 14, 2014; Revised March 09, 2014; Accepted March 15, 2014; Published Online April 09, 2014

\section{Original Article}

\begin{abstract}
Purpose: The aim of this study was to evaluate dosimetric differences between pencil beam convolution (PBC) algorithm and anisotropic analytical algorithm (AAA) calculations in patients with lung and esophageal cancers. Methods: The existing plans calculated with $\mathrm{PBC}$ for 60 patients treated in 2012 were recalculated with AAA maintaining the same beam geometry and dose monitor units. For these plans, dose prescription ranges were $41.4 \mathrm{~Gy}$ to $56.0 \mathrm{~Gy}$ for esophageal cancers and $50.0 \mathrm{~Gy}$ to 64.0 Gy for lung cancers. Dosimetric variables were the 95\% PTV coverage, mean PTV dose, maximum spinal cord dose, lung V5Gy, and lung V20Gy. Results: The 95\% PTV coverage's for both lung and esophageal tumors were reduced when recalculated with AAA. Maximum spinal cord doses for lung cancer patients were reduced by $0.7 \mathrm{~Gy}$ and by $0.3 \mathrm{~Gy}$ for esophageal cancer patients on AAA. On the other hand, lung V5Gy had 3.5\% increase for both lung and esophageal cancer patients on AAA, whereas lung $V_{20 G y}$ increased by $1.5 \%$ also on AAA for esophageal cancer patients. Conclusion: These clinical results confirm the differences between AAA and PBC algorithms as observed in phantom dosimetric studies, and give an indication of the clinical implications of changing from one calculation algorithm to another.
\end{abstract}

Keywords: Anisotropic Analytical Algorithm; Pencil Beam Convolution; Lung Cancer; Organs At Risk (OAR)

\section{Introduction}

Radical radiotherapy requires the calculated absorbed dose to be delivered accurately in order to achieve tumor control. ${ }^{1,2}$ In terms of treatment planning calculations; uncertainty in the dose calculation should therefore be kept to a minimum in order to achieve this maximal tumor control. The lung being a heterogeneous tissue creates difficulties in this regard. ${ }^{3}$ With the exception of Monte-Carlo simulation, which has been shown to be closest to reality for dose calculation

Corresponding author: Emmanuel Amankwaa-Frempong; Directorate of oncology, Komfo Anokye Teaching Hospital, P.O. BOX KS 1934, Kumasi, Ghana.

Cite this article as:

Amankwaa-Frempong E, Vernimmen F, Blay S, Ezhilalan R. Irradiation of lung and esophagus tumors: A comparison of dose distributions calculated by anisotropic analytical algorithm and pencil beam convolution algorithm, a retrospective dosimetric study. Int J Cancer Ther Oncol 2014; 2(2):020210.

DOI: $10.14319 /$ ijcto.0202.10 and distribution, other treatment planning algorithms such as Pencil Beam Convolution (PBC), Collapsed Cone and Anisotropic Analytical Algorithm (AAA) may not be that accurate. ${ }^{4}$ However, due to long calculation time with Monte-Carlo makes it impracticable to be used routinely in the current treatment planning systems. ${ }^{4}$

The PBC as documented in earlier findings performs poorly in calculating dose to tumors in and around the lungs. ${ }^{5-7}$ It does its calculations of dose distributions along a ray of line from the beam source (pencil beam). ${ }^{8}$ To account for differences in attenuation, there are corrections to each pencil beam that are obtained by a correction factor. ${ }^{9}$ These corrections are done in terms of calculation with respect to a point. ${ }^{9}$ The dose from the adjacent pencil beams is left out in the calculation leading to inconsistencies in dose determination of large tumors in homogeneities. ${ }^{10}$ 
The development of a superposition-convolution method known as AAA, has been shown to be more than accurate than $\mathrm{PBC}$ in photon dose calculations. ${ }^{11}$, 12 Because the AAA algorithm takes into account lateral scattering it calculates photon beams interactions in regions of complex tissue heterogeneities better. ${ }^{13}$ The AAA is known to be faster (reduces computational times). This algorithm has been incorporated in the Eclipse (Varian Medical Systems, Palo Alto, CA) integrated treatment planning system (TPS). ${ }^{14}$

The radiotherapy department of the Cork University Hospital (CUH) in the Republic of Ireland has been using Pencil Beam Convolution for the 3D-planning. Recently, as part of the implementation of intensity modulated radiation therapy (IMRT), the planning system was upgraded in order to use the AAA for dose calculations. In this study, we have performed a dosimetric comparison of $\mathrm{PBC}$ versus AAA dose calculations for a cohort of lung and esophageal cancer patients treated within the period of January-December, 2012. This study was approved by the ethical committee of CUH.

\section{Methods and Materials}

Patients: The department in CUH renders a radiotherapy service to a wide spectrum of cancer patients in Cork and the surrounding counties of Kerry, Waterford and South Tipperary. The department works in close collaboration with the departments of surgery, medical oncology, hematology, histopathology and radiology. A total of 237 lung and esophageal cancer patients were seen and treated during the period from January to December 2012. Of these, 60 patients were treated with radical intent for localized disease, with 31 lung cancer patients and 29 esophageal cancer patients. This study population consisted of 23 females and 38 males with respective age ranges of 50 - 86 years and $51-84$ years.

CT Simulation: The CT scanner used for simulation was a Siemens Somatom sensation, 40-slice helical scanner. Slice thickness was $3 \mathrm{~mm}$. Scans were obtained from the cricoid cartilage to the superior aspect of the L2 vertebra. Patients were positioned supine with arms immobilized above the head and their legs on a knee support.

Treatment plans: The software within our treatment planning system is Eclipse version 11.0, calculation grid size 2.5 $\mathrm{mm}$. For lung tumors gross tumor volume (GTV) was grown isotropically by $8 \mathrm{~mm}$ to produce the clinical target volume (CTV), and then edited taking into account likely patterns of spread to create the final CTV. Margins of $7 \mathrm{~mm}$ axially and $12 \mathrm{~mm}$ longitudinally are added to create the planning target volume (PTV). For esophageal cancers expanding the GTV by $2 \mathrm{~cm}$ longitudinally and $1 \mathrm{~cm}$ axially produced a CTV. The superior and inferior slices were edited. The CTV was grown isotropically by $5 \mathrm{~mm}$ axially and $10 \mathrm{~mm}$ superior inferiorly to produce the PTV. The existing PBC treatment plans for the 60 lung and esophageal cases were retrieved. These plans used beam energies of $6 \mathrm{MV}$ and $15 \mathrm{MV}$. Dose to the normalization point ranged from $41.4 \mathrm{~Gy}$ to $56.0 \mathrm{~Gy}$ for esophageal cancers and $50.0 \mathrm{~Gy}$ to $64.0 \mathrm{~Gy}$ for lung cancers. On the average two posterior oblique beams and one anterior beam arrangement was used for esophageal cancers. Three-field conformal plan was used for lung cancers and contralateral lung beams were avoided. For each patient, using the same beam configuration and original dose monitor units, dose distributions were recalculated with AAA. The following dosimetric variables were recorded: 95\% PTV coverage, maximum dose to PTV, mean dose to PTV, volume of lung covered by 5 Gy dose of radiation ( $V_{5 \mathrm{~Gy}}$ ) of lung, volume of lung covered by $20 \mathrm{~Gy}$ dose of radiation ( $\mathrm{V}_{20 \mathrm{~Gy}}$ ) of lung, and maximum spinal cord dose. All the lung volumes excluded the PTV.

Analysis: Statistical analysis was performed using STATA version 10 . Wilcoxon signed-rank test was used to determine the difference between $\mathrm{PBC}$ and AAA. When the $\mathrm{p}$-value was less than 0.05 the difference was deemed significant.

\section{Results}

Planning Target Volume: In this study, PTV volume ranged from $64.2 \mathrm{~cm}^{3}$ to $954.4 \mathrm{~cm}^{3}$. For lung tumors from (Table 1) the maximum and mean doses to the PTV as calculated with AAA were all lower as compared to PBC dose calculations. With a respective difference of $1.2 \mathrm{~Gy}(\mathrm{p}<0.001)$ and $2.2 \mathrm{~Gy}$ $(\mathrm{P}<0.03)$ observed. The 95\% PTV coverage was also lower $(84.5 \%)$ with AAA, with a difference of the median values of $10.5 \%$ as compared to PBC $(\mathrm{p}<0.001)$.

For esophageal tumors from (Table 2), the maximum and mean doses to the PTV on AAA are also significantly ( $\mathrm{p}<$ 0.001) lower although these differences are small compared to the lung values (0.5 Gy and $0.4 \mathrm{~Gy}$, respectively). The $95 \%$ PTV coverage was $89.5 \%$ with AAA with a difference for the median 95\% PTV coverage of $5.5 \%(\mathrm{p}<0.001)$.

Organs at risk: For lung cancer patients, (Table 3), the maximum spinal cord doses, although within clinical tolerance for the two algorithms, were significantly different with values $42.8 \mathrm{~Gy}$ and $42.1 \mathrm{~Gy}$ for $\mathrm{PBC}$ and $\mathrm{AAA}$, respectively ( $p=0.003$ ). The lung $\mathrm{V}_{5 \mathrm{~Gy}}$ was $42.5 \%$ for $\mathrm{PBC}$ and $46.0 \%$ for AAA, ( $<<0.001)$. Lung V20Gy was $21.0 \%$ for both AAA and PBC. For esophageal cancer patients, (Table 4), the maximum spinal cord dose was 29.1 Gy on PBC and 28.8 Gy on AAA ( $p=0.002)$. The lung $V_{5 G y}$ were $45 \%$ and $48.5 \%$ on PBC and AAA, respectively ( $\mathrm{p}<0.001)$. The lung $\mathrm{V}_{20 \mathrm{~Gy}}$ recorded $18.5 \%$ and $19.0 \%$ for PBC and AAA, respectively. 


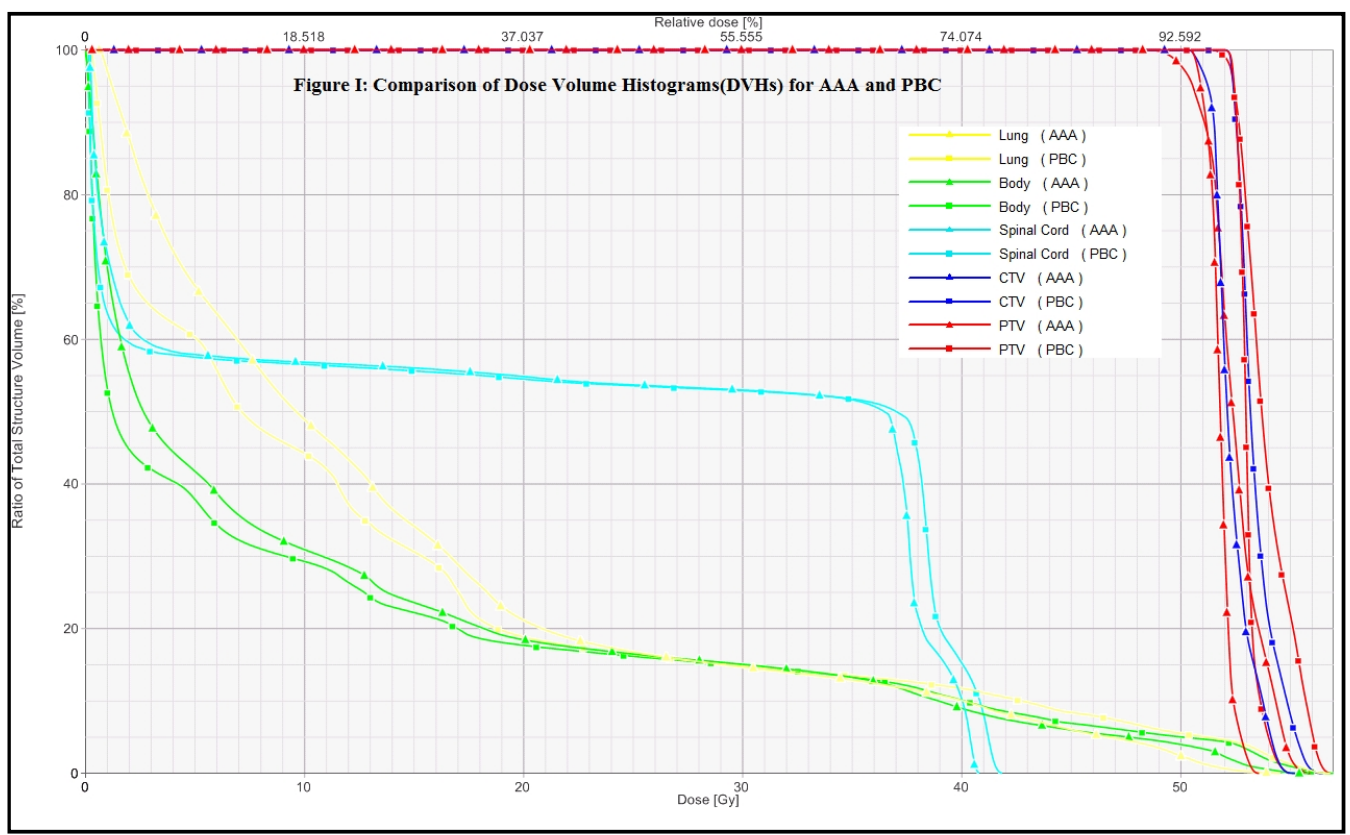

FIG. 1: Comparison of dose volume histograms (DVHs) for AAA and PBC

TABLE 1: Maximum and mean doses to PTV (in Gy) as well as 95\% PTV coverage (in percentage) for 31 lung cancer patients.

\begin{tabular}{cccc}
\hline \hline Parameter & PBC & AAA & p value \\
\hline Maximum dose PTV (Gy) & $63.3(59-64.4)$ & $62.1(58-63.9)$ & $<0.001$ \\
95\% PTV coverage (\%) & $95(95-95)$ & $84.5(81.2-86.3)$ & $<0.001$ \\
Mean PTV dose (Gy) & $60.2(56.0-60.3)$ & $58.0(53.9-58.7)$ & $<0.001$ \\
\hline \hline
\end{tabular}

TABLE 2: Maximum and mean doses to PTV (in Gy) as well as 95\% coverage (in percentage) for 29 esophageal cancer patients.

\begin{tabular}{cccc}
\hline \hline Parameter & PBC & AAA & p value \\
\hline Maximum dose PTV (Gy) & $44.3(43.8-54.4)$ & $43.8(43.3-53.2)$ & $<0.001$ \\
95\% PTV coverage (\%) & $95(95-95)$ & $89.5(88.1-90.8)$ & $<0.001$ \\
Mean PTV dose (Gy) & $41.6(41.5-50.8)$ & $41.2(40.9-50.0)$ & $<0.001$ \\
\hline \hline
\end{tabular}

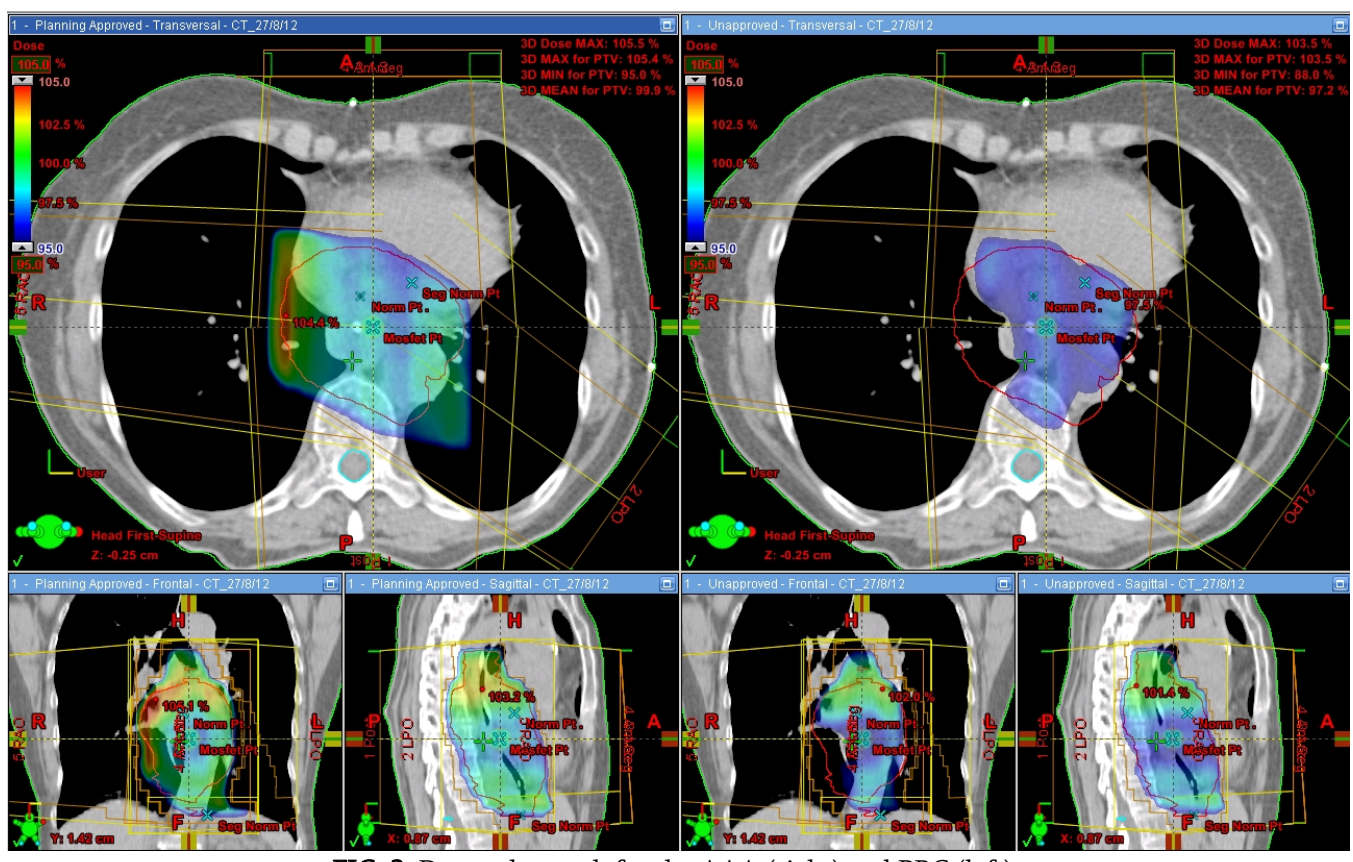

FIG. 2: Dose color wash for the AAA (right) and PBC (left). 
TABLE 3: Maximum dose to the cord, V5Gy, and $\mathrm{V}_{20 G \mathrm{G}}$ calculated on PBC and AAA plans for 31 lung cancer patients.

\begin{tabular}{cccc}
\hline \hline Parameter & PBC & AAA & P value \\
\hline Maximum cord dose (Gy) & $42.8(22.8-44.8)$ & $42.1(29-44.5)$ & 0.003 \\
Lung V20Gy (\%) & $21.0(15.0-27.5)$ & $21.0(15.0-27.5)$ & 0.405 \\
Lung V5Gy (\%) & $42.5(31.0-52.5)$ & $46.0(35.0-52.5)$ & $<0.001$ \\
\hline \hline
\end{tabular}

TABLE 4: Maximum dose to the cord, V5Gy, and V20Gy calculated on PBC and AAA plans for 29 esophageal cancer patients.

\begin{tabular}{cccc}
\hline \hline Parameter & PBC & AAA & P value \\
\hline Maximum cord dose (Gy) & $29.1(22.1-33.4)$ & $28.8(22.1-33.0)$ & 0.002 \\
Lung V20Gy (\%) & $18.5(11.2-27.5)$ & $19.0(11.0-26.2)$ & 0.008 \\
Lung V5Gy (\%) & $45.0(33.7-52.5)$ & $48.5(38.0-57.5)$ & $<0.001$ \\
\hline \hline
\end{tabular}

\section{Discussion}

Several studies have shown that in conventional 3-D planning AAA calculates dose distributions in a heterogeneous medium more accurately than PBC. Gagne and Zavgorodni 15 demonstrated this in the vertical water-lung interface phantom. ${ }^{15}$ Fogliata et al. also found that AAA calculations were satisfactory in terms of reproducing measured data for open and wedged beams as compared with PBC. ${ }^{16}$ In using a phantom, Cranmer-Sargison et al. ${ }^{17}$ was able to demonstrate an over-prediction of the dose to the lung portion of the phantom for AAA calculations. Carrasco et al. ${ }^{18}$, in comparing dose calculation algorithms in phantom with lung equivalent heterogeneities under conditions of lateral disequilibrium, showed that AAA was better for calculations in the lungs.

This study showed an over-estimation by PBC in calculating dose to lung and esophageal PTV's of $10.5 \%$ for lung tumors and $5.5 \%$ for esophageal tumors compared to AAA. This finding is consistent with the investigation of Ronde and Hoffman ${ }^{19}$, in which PBC overestimated the lung dose by 6 $10 \% .{ }^{19}$ Herman et al ${ }^{20}$, in evaluating PBC and AAA in lung irradiation also found a $10 \%$ over estimation of dose to the PTV by PBC. In that study, 20 treatment plans from PBC were recalculated with AAA, retaining identical beam arrangements, photon beam fluencies and dose monitor units. The PBC plans designed to give $60 \mathrm{~Gy}$, delivered 51.6 Gy when re-calculated with the AAA corresponding to $14 \%$ overestimation. ${ }^{20}$ In another study by Ueki et al. ${ }^{21}$, eighty-three patients with non-small-cell lung cancer (NSCLC) underwent stereotactic body radiation therapy (SBRT). AAA and PBC were compared to evaluate the dose-volume parameters under different heterogeneity corrections. The 95\% PTV coverage for $\mathrm{PBC}$ was $7 \%$ higher than AAA. Xiao et al. ${ }^{22}$ in the RTOG 0236 protocol evaluated twenty patients, comparing $\mathrm{PBC}$ plans after recalculating them on $\mathrm{AAA}$, and showed a decrease of $10 \%$ in the coverage of the $95 \%$ volume of the PTV. ${ }^{22}$

The radio therapeutic management of cancers has to strike a balance between sufficient dose to the target volume in order to get a cure on the one hand and a safe dose to the organs at risk on the other hand. Although the dose to the spinal cord in this study was different, both dose levels were clinically safe. AAA calculations underestimated the spinal cord dose for esophageal tumors and for lung cancer, by $1.0 \%$ and $1.6 \%$ between the two algorithms respectively, but both remained within clinical tolerance. Ronde and Hoffman, who compared AAA and PBC treatment plans in lung geometry found that AAA underestimated the spinal cord dose by as much as 7\%. ${ }^{19}$ In another study, however, comparing Monte Carlo simulation and AAA, the AAA was observed to overestimate the spinal cord dose by only $0.2 \%{ }^{23}$ These findings are re-assuring in terms of doses to the spinal cord all remaining within clinical tolerance.

In terms of the lung radiation tolerance parameters ( $V_{5 G y}$ and $\mathrm{V}_{20 \mathrm{~Gy}}$ ) we observed no differences between PBC and AAA for lung $\mathrm{V}_{20 \mathrm{~Gy}}(21.0 \%)$, in both esophageal and lung cancers. The difference in integral lung dose is reflected in the difference of the $\mathrm{V}_{5 \mathrm{~Gy}}$ values between AAA and PBC. This difference increases slightly for increasing volumes of PTV's (Figure 1), with $V_{5 G y}$ recordings showing a 3.5\% difference for lung and esophageal cancers between $\mathrm{PBC}$ and AAA, all been highest with AAA. The $\mathrm{V}_{5 \mathrm{~Gy}}(46 \%)$ and $\mathrm{V}_{20 \mathrm{~Gy}}(21 \%)$ is consistent with findings of Kroon et al. ${ }^{24}$, who had $46.6 \%$ and $19.3 \%$ for $V_{5 G y}$ and $V_{20 G y}$, respectively for lung tumors also calculated on AAA. ${ }^{24}$ The difference in mean PTV doses between PBC and AAA for lung and esophageal cancers were $4 \%$ and $1 \%$, respectively. Fotina et al. ${ }^{25}$ in comparing collapsed cone and Monte-Carlo algorithm observed less than 3\% difference between the two algorithms. The Acuros XB calculation algorithm for photon beams has been shown to be more accurate than AAA ${ }^{26-28}$, and it has also been observed to have a relatively lower PTV dose as compared to AAA ranging from $2-4 \% .^{26}$ This shows that there is not much difference between the algorithms in terms of mean PTV dose. Therefore, based on mean PTV doses, tumor control may be similar irrespective of the algorithm used. However, caution is warranted when treating lung tumors especially when the PTV volume is large and high energy beams are used.

\section{Conclusion}

The results from our study have shown that the differences reported in other studies with $\mathrm{AAA}$ and $\mathrm{PBC}$ algorithm in 
terms of PTV coverage and organs at risk also occurred in our study population of a random group of esophageal and lung cancer patients. To avoid overestimation, it is recommended to use the AAA algorithm in the treatment planning instead of $\mathrm{PBC}$, especially for the lung tumors. In adopting $\mathrm{AAA}$ as a new algorithm into a clinical treatment planning practice, a good understanding of the effects and potential consequences by oncologists is important.

\section{Conflict of interest}

The authors declare that they have no conflicts of interest. The authors alone are responsible for the content and writing of the paper.

\section{Acknowledgements}

The authors would like to thank the dosimetrists and physicists in the planning section of the department of Radiation Oncology in Cork University hospital for their assistance and support.

\section{References}

1. Brahme A. Dosimetric precision requirements in radiation therapy. Acta Radiol Oncol 1984; 23:379-91.

2. International Commission on Radiation Units and Measurements. Determination of absorbed dose in a patient irradiated by beams of $\mathrm{X}$ or gamma rays in radiotherapy procedures.1976; ICRU Report 24.

3. Metcalfe PE, Wong TP, Hoban PW. Radiotherapy $\mathrm{X}$-ray beam inhomogeneity corrections: the problem of lateral electronic disequilibrium in lung. Australas Phys Eng Sci Med 1993; 16:155-67.

4. Ma CM1, Mok E, Kapur A, et al. Clinical implementation of a Monte Carlo treatment planning system. Med Phys 1999; 26:2133-43.

5. Krieger T, Sauer OA. Monte Carlo- versus pencil-beam-/collapsed-cone-dose calculation in a heterogeneous multi-layer phantom. Phys Med Biol 2005; 50:859-68.

6. Sikora M, Muzik J, Sohn M, et al. Monte Carlo vs. Pencil Beam based optimization of stereotactic lung IMRT. Radiat Oncol 2009; 4: 64.

7. Tachibana M, Noguchi Y, Fukunaga J, et al. Influence on dose calculation by difference of dose calculation algorithms in stereotactic lung irradiation: comparison of pencil beam convolution (inhomogeneity correction: batho power law) and analytical anisotropic algorithm. Nihon Hoshasen Gijutsu Gakkai Zasshi 2009; 65:1064-72.

8. Storchi PR, van Battum LJ, Woudstra E. Calculation of a pencil beam kernel from measured photon beam data. Phys Med Biol 1999; 44:2917-28.
9. Hurkmans C1, Knoos T, Nilsson P, et al. Limitations of a Pencil Beam approach to photon dose calculations in the head and neck region. Radiother Oncol 1995; 37:74-80.

10. Robinson D. Inhomogeneity correction and the analytic anisotropic algorithm. J Appl Clin Med Phys 2008; 9: 2786.

11. Ulmer W, Kaissl W. The inverse problem of a Gaussian convolution and its application to the finite size of the measurement chambers/detectors in photon and proton dosimetry. Phys Med Biol 2003; 48:707-27.

12. Ulmer W, Harder D. A Triple Gaussian Pencil Beam Model for Photon Beam Treatment Planning, Z. MedPhys 1995; 5:25-30.

13. Ulmer W, Brenneisen W. Application of an Analytical Pencil Beam Model to Stereotactic Radiation Therapy Planning. Journal of Radiosurgery 1998; 1:169-76.

14. Sievinen J, Ulmer W, Kaissl W. AAA photon dose calculation model in Eclipse. Palo Alto (CA): Varian Medical Systems; 2005.

15. Gagne IM, Zavgorodni S. Evaluation of the analytical anisotropic algorithm in an extreme water-lung interface phantom using Monte Carlo dose calculations. J Appl Clin Med Phys 2006; 8:33-46.

16. Fogliata A, Nicolini G, Vanetti E, et al. Dosimetric validation of the anisotropic analytical algorithm for photon dose calculation: fundamental characterization in water. Phys Med Biol 2006; 51:1421-38.

17. Cranmer-Sargison G, Beckham WA, Popescu IA. Modelling an extreme water-lung interface using a single pencil beam algorithm and the Monte Carlo method. Phys Med Biol 2004; 49: 1557-67.

18. Carrasco P, Jornet N, Duch MA, et al. Comparison of dose calculation algorithms in phantom with lung equivalent heterogeneities under conditions of lateral disequilibrium. Med Phys 2004; 31:2899-911.

19. Ronde HS, Hoffmann L. Validation of Varian's AAA algorithm with focus on lung treatments. $A c^{-}$ ta Oncol 2009; 48: 209-15.

20. Herman Tde L, Hibbitts K, Herman T, Ahmad S. Evaluation of pencil beam convolution and anisotropic analytical algorithms in stereotactic lung irradiation. JMed Phys 2011; 36: 234-8.

21. Ueki N, Matsuo Y, Shibuya K, et al. Differences in the dose-volume metrics with heterogeneity correction status and its influence on local control in stereotactic body radiation therapy for lung cancer. J Radiat Res 2013; 54: 337-43.

22. Xiao Y, Papiez L, Paulus R, et al. Dosimetric evaluation of heterogeneity corrections for RTOG 0236: stereotactic body radiotherapy of inoperable stage 
I-II non-small-cell lung cancer. Int J Radiat Oncol Biol Phys 2009; 73:1235-42.

23. Gagne IM, Ansbacher W, Zavgorodni S, et al. A Monte Carlo evaluation of RapidArc dose calculations for oropharynx radiotherapy. Phys Med Biol 2008; 53: 7167-85.

24. Kroon PS, Hol S, Essers M. Dosimetric accuracy and clinical quality of Acuros XB and AAA dose calculation algorithm for stereotactic and conventional lung volumetric modulated arc therapy plans. Radiat Oncol 2013; 8:149.

25. Fotina I, Kragl G, Kroupa B, et al. Clinical comparison of dose calculation using the enhanced collapsed cone algorithm vs. a new Monte Carlo algorithm. Strahlenther Onkol 2011; 187:433-41.

26. Rana S. Clinical dosimetric impact of Acuros XB and analytical anisotropic algorithm (AAA) on real lung cancer treatment plans: review. Int J Cancer Ther Oncol 2014; 2: 02019.
27. Lu L. Dose calculation algorithms in external beam photon radiation therapy. Int J Cancer Ther Oncol 2013; 1:01025.

28. Rana S, Rogers K, Pokharel S, Cheng C. Evaluation of Acuros XB algorithm based on RTOG 0813 dosimetric criteria for SBRT lung treatment with RapidArc. J Appl Clin Med Phys 2014; 15:4474.

29. Fogliata A, Nicolini G, Clivio A, Vanetti E, Cozzi L. Critical appraisal of Acuros XB and Anisotropic Analytic Algorithm dose calculation in advanced non-small-cell lung cancer treatments. Int J Radiat Oncol Biol Phys 2012; 83:1587-95. 